\title{
Characteristics of ex-racing greyhounds in New Zealand and their impact on rehoming
}

\author{
JB Thomas ${ }^{1,2 *}$, NJ Adamsi and MJ Farnworth ${ }^{1,3}$ \\ 1 Animal Welfare and Biodiversity Research Group, Environmental and Animal Sciences, Unitec Institute of \\ Technology, Private Bag 92025, Auckland, New Zealand \\ 2 Currently: School of Psychology, University of Waikato - Tauranga, Private Bag 12027, Tauranga 3143, New Zealand \\ 3 Currently: School of Biological Sciences, A426 Portland Square, Plymouth University, Drake Circus, Devon, PL4 8AA, \\ United Kingdom \\ *Contact for correspondence and requests for reprints: $\underline{\text { Julia@foxx.co.nz }}$
}

Abstract (250 words)

A small proportion of greyhounds surplus to the racing industry are entered into specialist rehoming organisations to be re-purposed as pets. Records of 835 greyhounds, from New Zealand Greyhounds as Pets (GAP), were used to investigate whether preadoption characteristics (age, sex, racing record, reason entered) and management factors (temperament test result, foster and trainer effects) had a bearing on rehoming success, and comparisons were made with shelter studies. Rehoming greyhounds as pets is very successful with $85.5 \%$ ultimately successfully rehomed. Only $2.9 \%$ fail as a result of failed adoptions, $11.6 \%$ fail the initial temperament test. Greyhounds were more likely than shelter dogs to pass an initial temperament test and be adopted, and less likely to be returned after 1 month. However, adopted greyhounds were just as likely as shelter dogs to be returned after 6 months. Logistic regression revealed the youngest age group $(<24$ months old) were more likely to pass the initial temperament test than older greyhounds. This age effect was not detectable when the adoption success of dogs subsequently available for rehoming was considered, but a sex effect was evident with females more likely to be successfully adopted than males. Whether or not a dog had raced had no significant effect on the likelihood of successful rehoming. 
27 Greyhounds passing the temperament test with a basic pass, were less likely to be successfully rehomed than greyhounds scoring a higher pass indicative of lower prey drive. Further investigation of the validity and reliablity of the temperament test is warranted.

\section{Introduction}

The greyhound racing industry produces substantial numbers of dogs that are not needed or suitable for racing (Colgan et al 2013). Acceptance of the the sport is changing as concern and awareness about the scale and method of destruction of surplus animals and the risk of injury arising from the sport, grow (Atkinson \& Young 2005; as reported in Colgan et al 2013 p 28; Madden 2010). Greyhounds have a life expectancy of 10-12 years (Fogle 2000), but an average racing career spans just 1.5 years with the average age of retirement in New Zealand being 3.37 years (Colgan et al 2013). This potentially allows an ex-racing greyhound to spend more than 8 years another role, and the rehoming of retired and surplus racing greyhounds as pets has increased in popularity (Lord et al 2007). The New Zealand Greyhounds As Pets (GAP) charity was established by the New Zealand Greyhound Racing Authority (NZGRA) in 2006 and aims to rehome greyhounds put forward by trainers and breeders in the 45 industry.

48 The success of greyhound adoptions through the GAP programme in Australia and New 49 Zealand, was evaluated by Elliott et al (2010) one-month post-adoption. Most 50 adoptions were successful (237/245) with a high proportion of owners $(91.1 \%)$ scoring

51 'very satisfied' in terms of the greyhound fulfilling their expectations as a pet. A 
52 significant association between 'realistic owner expectations' and decreased likelihood

53 a dog would be returned after adoption, has been reported for dogs rehomed from

54 shelters (Marston et al 2005).

55 The primary reason dogs are returned to adoption agencies is reported to be problem

56 behaviour, accounting for between $58.6 \%$ (Diesel et al 2008b) and 89.7\% (Wells \&

57 Hepper 2000) of returns to shelters. Although based on a small number of failed 58 adoptions, Elliott et al (2010) similarly reported most greyhounds were returned due to 59 behaviour related problems.

Behavioural problems most likely to put adoptions at risk are related to aggression, separation anxiety, hyperactivity, noisiness and incompatibility with other pets (Diesel et al 2008b; Elliott et al 2010; Marston \& Bennett 2003). Previous studies have shown associations between the development of particular behaviour problems and a dog's breed (Duffy et al 2008) age, sex, background (McGreevey \& Masters 2008; Wells \& Hepper 2000) early experiences (McMillan et al 2011) and training methods (Blackwell et al 2008; Thompson et al 2010).

Investigating the rehoming of ex-racing greyhounds, provides a unique opportunity to research factors associated with known pre-adoption histories of individuals from a single breed, not bred or held primarily as companion animals. We characterise descriptively the attributes and history of dogs entering the programme and investigate whether there is a significant association between selected pre-adoption factors

74 (including age, sex, racing record, reason for entry, trainer and temperament test result) 75 and the likelihood of successfully rehoming greyhounds bred specifically for racing. 


\section{Methods}

Details of all greyhounds entering the New Zealand GAP programme between 01 April 2010 and 31 March 2014 were used unless there was no reported outcome by 01 September 2014 (i.e. the dog was still waiting to be assessed or adopted).

Dogs entered the GAP programme either via a trainer or welfare admission. Greyhounds entered under a welfare admission had either been removed from the owner by GRNZ, or surrendered to, or removed by an animal welfare organisation.

Information was extracted from the New Zealand GAP database including the reported reason for entering the dog into GAP, the dog's age, sex, health/injury status, temperament test result, racing history, foster record, and rehoming outcome. The entry reasons given were grouped into 5 categories, and reported injuries and health issues were attributed to one of 8 categories (Table 1). Dogs were allocated to one of three age groups: 'young' (5 - 24 months old), 'adult' (25 -66 months old), and 'senior' (67 - 146 months old) (Table 1).

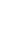

A temperament test administered to all greyhounds entering GAP determined whether the dog was made available for adoption. The GAP New Zealand temperament test is based on the Australian Greyhound Behavioural Assessment. The test is performed no sooner than the third day after arrival at the rehoming kennels and comprises assessments of the dog's behaviour (including fearfulness, anxiety, arousal levels, affiliative/aggressive behaviours, shyness/boldness, leash manners, sociability and noisiness), in relation to eight assessment items (Table 8). Each element of the assessment item is scored out of three, one is a pass, two requires reassessment and/or 
102 time in a foster home, and three is a fail. A dog must score a one for every element of

103 the assessment item to be considered for adoption, with the exception of the 'reaction

104 to cat' element, whereby a score of one results in a 'pass with cats' (TTP+) and a score

105 of two results in a basic pass (TTP). Scores for each assessment element have

106 corresponding behaviours, for example a score of 'one' for the 'reaction to cat' element

107 includes "tail relaxed or wagging, dog solicits polite interaction, dog easily distrated by

108 handler"; a score of 'three' includes "signs of high prey drive - shaking, trembling,

109 fixed stare, lunges towards cat, barking, salivating, cannot be distracted despite multiple

110 attempts".

112 Dogs were assessed as acceptable for homing with humans and other dogs (including 113 small dogs) if they passed the temperament test at the basic level (TTP). Greyhounds 114 that passed with a (TTP+) were considered potentially suitable for a home that included 115 cats. A TTP+ was considered to be indicative of lower prey drive (i.e. the innate 116 disposition of a canid to locate, chase and capture prey) relative to a TTP.

118 All dogs that passed the temperament test were desexed prior to being fostered or 119 adopted.Some greyhounds were fostered by GAP volunteers before being adopted, 120 allowing the organisation to further assess and prepare dogs for adoption. The duration 121 of each foster placement was not consistently reported in the GAP database, hence only 122 the number of foster placements (if any) was recorded for each dog. In some cases 123 greyhounds were recorded as being 'fostered to adopt'. This designation was used when 124 volunteers were unsure about the suitability of a greyhound, or when volunteers 125 provided a foster home for GAP dogs with an intention of possibly adopting that dog. 126 If the GAP database reported 'foster to adopt', and the person fostered and subsequently 
adopted the dog, the date of adoption was recorded as the 'foster to adopt' date. If the greyhound was returned to the GAP kennel and was subsequently rehomed to a different person, the record was counted as a normal foster placement rather than a failed adoption.

Greyhounds that passed the temperament test and were adopted were considered successfully rehomed. If a dog was subsequently returned but successfully readopted, the rehoming was still considered 'ultimately successful'. Greyhounds could 'fail' at two stages during the rehoming process: 1) prior to being made available for adoption, either as a result of the temperament test outcome, or displaying unacceptable behaviour in a foster home; or 2) if returned after being adopted. Dogs that 'failed' at stage 1 and those deemed unsuitable for re-adoption after being returned (stage 2) were humanely destroyed by a veterinarian.

In addition to information held by GAP, racing records for individual greyhounds (i.e. number of race starts and podium finishes) were retrieved from the GRNZ public website (www.thedogs.co.nz). A performance score was calculated by determining the total number of podium finishes (firsts, seconds and thirds) as a percentage of the total number of race starts for each dog. Dog's performance scores were sorted into low, medium and high performance groups, and the number of races raced per dog was similarly sorted into low, medium and high categories, based on percentiles of the distribution of results (Table 1). We classified a greyhound as 'unraced' when recorded as unraced in the GAP database and having no racing record on the GRNZ web site. 
151 We used binary logistic regression to examine the main effects of year of entry, age 152 group, sex, entry reason, racing history (raced or unraced) on whether dogs passed or

153 failed the temperament test and therefore whether they were considered for adoption.

154 This analysis was completed on all 825 dogs entering the GAP programme. All analyses

155 were performed using a backward stepwise elimination of non-significant independent

156 variables. To manage the number of independent factors or variables, we did not 157 consider specific racing performance, possible trainer or foster effects or the specific 158 injury or health issues associated with particular dogs in this analysis. These data are 159 presented descriptively.

161 We then completed a similar analysis for dogs that initially passed the temperament test $162(\mathrm{n}=738)$. This allowed us to explore the possible effect of temperament test (either 163 basic pass (TTP) or 'pass with cats' (TTP+)), along with year of entry, sex, entry reason, 164 and racing history (raced or unraced), on ultimate adoption success. We repeated this 165 analysis on a slightly smaller group that excluded nine dogs $(n=729$ dogs $)$ that passed 166 the temperament test, but were initially fostered but then not put up for adoption 167 because of unresolved behavioural issues. We followed this by exploring the same 168 main effects, but in regard of whether dogs were successfully adopted at the first 169 attempt at rehoming or not. As previously we ran these analyses for all dogs that 170 initially passed the temperament test $(n=738)$ or the slightly smaller group $(n=729)$ 171 (see above).

173 We also conducted a binomial logistic regression to explore the possible association of 174 trainer, age group, sex, racing history and year of entry, on temperament test outcome 175 (fail or pass). A total of 145 different trainers entered dogs into GAP, so we restricted 
176 our analysis to those dogs from trainers that entered 25 dogs or more into the GAP 177 programme.

\section{Results}

\section{Descriptive statistics}

181 The annual number of greyhounds entering GAP New Zealand increased by $23.9 \%$ between $2011(n=197)$ and $2014(n=244)$. A total of 835 greyhounds entered the

183 GAP programme between April 2010 and March 2014, of which 714 (85.5\%) were 184 successfully rehomed. A total of 605 (72.5\%) were successful the first time they were 185 adopted and a further 109 (13.0\%) were successfully re-adopted after being returned 186 (Figure 1). Of the $14.5 \%(121 / 835)$ of dogs that ultimately failed to be rehomed $11.6 \%$ 187 (97/835) failed the initial temperament test, and $2.9 \%(24 / 835)$ were destroyed 188 following return from adoption or foster (Figure 1).

190 Of the $738(88.4 \%)$ dogs that passed the initial temperament test $264(35.8 \%)$ were 191 initially fostered, after which 9 dogs failed, leaving 729 dogs available for adoption. A 192 total of $349(47.9 \%)$ of these dogs were homed between one and two months of entering 193 GAP and 659 (90.4\%) within four months. Median length of stay in rehoming kennels 194 (between entry and first adoption) was 27 days (range 0 to 378 days). A total of 124 195 (17\%) dogs were returned after their first adoption, mostly (70 dogs, 56.5\%) within 196 four months of initial adoption (Figure 2). The median number of days between 197 adoption and return was 89 days (range 0 to 1137 days).

199 Median age at entry to the GAP programme was 44 months (range 5 - 146 months, $25^{\text {th }}$ $200-75^{\text {th }}$ percentile: $31-54$ months respectively). A total of $77.4 \%$ of dogs were classified 
201 as adults of 25 to 66 months of age (Table 1). There was a suggestion that young dogs 202 ( $<25$ months old) were more likely to pass the temperament test than adults and/or 203 seniors (Table 2) (see analysis below).

205 The number of male and female greyhounds entering GAP was similar (Table 1). 206 However slightly more female dogs $(88.1 \%)$ were successfully adopted compared with 207 males (83.1\%) (Table 3). Entry reasons were recorded for 738 of $835(88.4 \%)$ 208 admissions. The most prevalent reason for entering a dog into the GAP programme was 209 the category 'age, retirement, end of racing career' (41.7\%, Table 1). Although dogs 210 were uniquely allocated to an entry reason category, classifications were not mutually 211 exclusive. A total of $14.1 \%$ of admissions were reported as being due to injury or health 212 concerns. However this is an underestimate as dogs from other 'entry reason' categories 213 (e.g. welfare) also had injuries and health issues. Records of the actual nature of injuries 214 indicated 196 of 835 (23.5\%) greyhounds entering the GAP programme had health 215 and/or injury issues (Table 1). The most prevalent identified injuries were to the foot 216 and hock followed by gracilis injuries (Table 1).

218 A minority (18.2\%) of greyhounds entering GAP were unraced (Table 1). There was a 219 suggestion that unraced dogs were more likely to pass the temperament test than raced 220 dogs (Table 2) but this effect was not significant (see regression analysis below). A 221 total of $81.8 \%(n=683)$ of greyhounds entering GAP had raced 1 or more times 222 (median: 47 starts per dog, range $1-177$ race starts, $25^{\text {th }}$ and $75^{\text {th }}$ percentiles 25 and 76 223 starts respectively, Table 1). The median performance of raced greyhounds, expressed 224 as the percentage of podium finishes to total number of race starts was $35.6 \%$ (range $2250 \%-100 \%, 25^{\text {th }}-75^{\text {th }}$ percentile: $25 \%-44 \%$, Table 1$)$. 
227 A total of $88.4 \%$ (Table 2) of greyhounds passed the initial temperament test with more 228 dogs passing with a TTP+ result (55\%) than a TTP (45\%). A total of $98.8 \%$ of TTP+ 229 dogs were ultimately successfully rehomed compared with $96.9 \%$ of dogs with TTP, 230 and fewer TTP+ dogs were returned after their first adoption than dogs with a TTP 231 result (13.6\% and $21.2 \%$ respectively, Table 3$)$.

232

233 Of the dogs that passed their initial temperament test $35.8 \%(n=264)$ were fostered at 234 least once before being adopted the first time (Table 1). A total of 46.8\% $(n=58)$ of 235 returned dogs were fostered before being rehomed a second time. The percentage of 236 fostered dogs that were not made available for a first or second adoption (i.e. were 237 destroyed following their foster placement) was $3.4 \%(\mathrm{n}=9)$ and $3.4 \%(\mathrm{n}=2)$ 238 respectively. Of the dogs that were not fostered, $18.1 \%(86 / 474)$ were returned after 239 their first adoption and of these $2.1 \%$ were not re-adopted (i.e. were destroyed). Of the 240 dogs that were fostered, $14.4 \%$ (38/264) were returned after their first adoption and $2412.0 \%$ of these were destroyed. A total of $56.1 \%(148 / 264)$ of fostered dogs had passed 242 the temperament test with a TTP result compared with $38.8 \%(184 / 474)$ of dogs that 243 were not fostered. Overall, $94.7 \%$ of greyhounds fostered before their first adoption 244 were successfully rehomed, compared with $97.9 \%$ of dogs that were not fostered first 245 (Table 3).

247 Five of 125 trainers $(2.8 \%)$ entered 25 dogs or more into the GAP programme. Two of 248 these trainers (labelled B and D, Table 4) entered substantially more younger dogs 249 (39.3\% and $60.0 \%$ respectively) than the other three (trainer A: $5.1 \%$, trainer C: $2.4 \%$ 250 and trainer E: 0\%). The sex, racing history and entry reason profiles of dogs entered, 
251 also varied among trainers (Table 4). Trainers B and D had 3.3\% of their dogs fail the 252 temperament test, compared with trainers A, C and E whom had 12.8\%, $19.5 \%$ and $25324 \%$ of their dogs fail respectively (Table 4), but these differences were not significant 254 (see below).

\section{Factors affecting temperament test pass or fail}

258 Our binary logistic regression performed to ascertain the main effects of year of entry, 259 age group, sex, entry reason and racing history (raced or unraced), on whether dogs 260 passed the temperament test and were considered for adoption, was statistically 261 significant $\left(\chi^{2}=23.468, \mathrm{df}=5, P<0.001\right)$. Of the five independent variables, only age 262 proved statistically significant (Table 5). Indicated by an odds ratio of less than 1, adult 263 and senior dogs were both significantly less likely to pass the temperament test 264 compared to young dogs (Table 5).

266 The binomial logistic regression performed to ascertain the main effects of trainer (with 26725 dogs or more entered into GAP programme), age group, sex, racing history (raced 268 or unraced) and year of entry on temperament test outcome, showed no significant main 269 effects.

\section{Factors affecting ultimate adoption success}

273 The analysis to ascertain the main effects of temperament test, year of entry, age group, 274 sex, entry reason and racing history (raced or unraced), on ultimate adoption success 275 was also statistically significant $\left(\chi^{2}=27.523\right.$, df $\left.=5, P<0.001\right)$. Temperament test 
276 pass type, sex of dog and year of entry proved to be significant main effects. Dogs 277 passing at the basic level temperament test (TTP) were less likely to successfully 278 rehome than those passing the higher test (TTP+) (Odds ratio: 0.328, $95 \%$ CI: 0.131279 0.820), females were more likely to be successfully adopted than males (Odds ratio: $2802.895,95 \%$ CI: $1.117-7.503)$, dogs entering the programme in 2014 were marginally 281 less likely to be successfully rehomed than dogs entering in $2011(0.177,95 \%$ CI: $2820.039-0.799$ ) (Table 6). When we repeated the analysis on our slightly smaller group of 283 dogs that were considered for adoption $(n=729$ after nine dogs destroyed following 284 their initial foster placement were removed) the model remained significant $\left(\chi^{2}=\right.$ $28520.069, \mathrm{df}=4, P<0.001)$, with $\operatorname{sex}(\mathrm{P}=0.01)$ and year of entry $(\mathrm{P}=0.028)$ effects 286 remaining, however the effect of temperament test pass disappeared.

\section{Factors affecting success at first adoption}

Models based on the sample of 738 dogs that passed the initial temperament test $\left(\chi^{2}=\right.$ $2919.644, \mathrm{df}=1, P=0.002$ ) or $729 \mathrm{dogs}$ (after nine dogs destroyed following their initial 292 foster placement were removed) $\left(\chi^{2}=7.359\right.$, df $\left.=1, P=0.007\right)$ were significant. 293 Success at first adoption was related only to the outcome of the temperament test for 294 both the model utilizing 738 dogs (Table 7) or that based on the slightly smaller sample $295 \quad(\mathrm{n}=729 \operatorname{dogs})(\mathrm{P}=0.007)$.

\section{Discussion}

299 Most greyhounds $(87.3 \%)$ entering the GAP programme pass an initial temperament 300 test and are rehomed. Shelter studies, which also include an initial temperament test, 
301 generally report a much lower initial rehoming rate (e.g. 21.3\% Marston et al (2004); $30227 \%$ Mornement et al (2010)). This is even when these figures are corrected for animals 303 reclaimed by owners, euthanised for health or welfare reasons or destroyed as a 304 'prohibited breed type' (e.g. 49.4\% calculated from Marston et al (2004)). This 305 relatively high success rate of the GAP programme is carried over into re-adoption 306 success of greyhounds returned to the programme after an initial failed adoption. Only $30712.1 \%$ of returned greyhounds were destroyed, compared to between $40 \%$ (Marston et al 2004) and 50\% (Patronek et al 1995) of returned shelter dogs.

310 Although greyhounds have a similar average length of stay in rehoming kennels prior 311 to adoption (median 27 days) compared to shelter dogs (e.g. 28 days Diesel et al 2007; 31223 days Zak et al 2015), most companion dogs will not have previously spent extensive 313 periods in a high-density kennel environment (Taylor \& Mills, 2007; Wells 2004). 314 Greyhounds on the other hand, have generally spent all their pre-adoption lives in 315 kennels. High density kennel housing of dogs has the potential to cause considerable 316 stress, and contribute to the development of behaviour problems (Diesel et al 2008b; 317 Marston et al 2004; Taylor \& Mills, 2007; Wells 2004) before and after adoption. Dogs 318 that are preconditioned to kennelling show decreased physiological signs of stress 319 compared with dogs that have not had prior habituation to a kennelled environment 320 (Rooney et al 2007). The fact that most greyhounds have been pre-conditioned to 321 kennelling, might contribute to their relatively high rehoming success compared with 322 dogs from shelters.

324 However, owners' attitudes cannot be discounted. Although they were not a component 325 of this work, evidence suggests that people are more likely to accommodate behaviours 
326 associated with a particular purebred dog (Coren 2000). For example, new owners are

327 likely to be more tolerant of a terrier that digs (or a greyhound that chases things), if

328 they specifically want that type of dog. Whereas new owners of shelter dogs might not

329 be as accepting of potentially undesirable behaviours.

331 Pet dogs relinquished to shelters are also likely to have existing behaviour problems

332 (Diesel et al 2008b; Marston et al 2004; Wells \& Hepper 2000), whereas greyhounds

333 entered into the GAP rehoming programme are potentially pre-selected for desirable 334 attributes. Only a small proportion (15.5\% (calculated from Colgan et al 2013)) of 335 surplus racing industry greyhounds are entered into the GAP programme. Although the 336 precise criteria used by trainers to select dogs to enter the programme is unknown and 337 appears to vary among trainers (Table 4), greyhound trainers pay a fee to enter dogs 338 into the rehoming programme and may elect not to submit dogs displaying undesirable 339 behaviours that would result in dogs failing the initial temperament test. Consistent 340 with this view, considerably fewer greyhounds failed the initial temperament test 341 (11.6\% (97/835)) compared with pet dogs entering rehoming shelters $(29.3 \%$ 342 (calculated from Marston et al 2004)). However, differences may also be explained in 343 part by the different criteria used to evaluate temperament between the different studies.

345 There were a variety of reasons given for entering dogs into the GAP programme and 346 a considerable age range. However, the most prevalent entry reason category was 347 related to 'retirement from racing', explaining at least in part the age profile of dogs 348 entered. The median age of dogs entered (3.7 years) was consistent with the reported 349 average age of retirement (Colgan et al 2013). 
352 Due to our study being an investigation of historical data, we were not able to examine

353 the validity, repeatability or inter-rater reliability of the GAP temperament test.

354 However, there was no significant effect of year of entry detected for temperament test 355 outcome (pass/fail), which might suggest the GAP temperament test has been relatively 356 consistent over time.

358 In this study, only dogs that passed the temperament test were actually placed for 359 potential adoption. Accordingly the assessment of the impact of temperament testing 360 on adoption rates could only be assessed for dogs that passed the temperament test, 361 either as a TTP or TTP+. The emphasis on prey-drive in the GAP two-tier temperament 362 test reflects the unique concerns associated with re-purposing ex-racing greyhounds 363 that have been trained to strengthen the 'chase' component of the predatory hunting 364 sequence. Dogs that passed the temperament test with TTP+, purportedly indicative of 365 lower prey drive relative to TTP, were more likely to be successfully rehomed 366 (ultimately). Further, greyhounds that achieved a TTP were more likely to be returned 367 after being adopted (21.2\% returned) than those that achieved TTP+ (13.6\% returned). 368 Although we can not confirm the validity of the temperament test's ability to determine 369 the level of prey drive, our results support the suggestion that higher prey drive might 370 be associated with increased risk of rehoming failure (Elliott et al 2010). However, 371 given the lack of evidence to confirm temperament tests accurately predict stable 372 behaviour over time (Bennett et al 2012), and the fact that $80 \%$ of greyhounds that fail 373 to be successfully rehomed fail at the initial temperament test stage, it would be 374 interesting to investigate the validity and reliability of the GAP temperament test. 
377 For those dogs that passed the temperament test, there was a year of entry effect on 378 ultimate rehoming success. Dogs returned in 2011, after their first adoption, were more 379 likely to be successfully readopted (only 1.2\% failed) than dogs returned in 2014 (6.5\% 380 failed). The reason(s) the GAP programme was less likely to successfully re-adopt 381 dogs in 2014, than it did in 2011 is not known. It could be associated with limited 382 funding and an increased target for the number of greyhounds rehomed each year (GAP 383 2014), effectively reducing resources (e.g. kennel space and time) able to be allocated 384 to re-adoption of returned greyhounds.

386 The proportion of greyhounds returned at one-month post-adoption (2.7\%) was similar 387 to that reported by Elliott et al (2010) (3.3\%), but somewhat less than shelter studies 388 (6.5\% in Northern Ireland (Wells \& Hepper 2000); 12.9\% in Melbourne shelters 389 (Marston et al 2005)). Diesel et al (2008b) reported a six-month post-adoption return 390 rate of $14.7 \%(662 / 4500)$ in the United Kingdom, which is similar to the $11.7 \%$ 391 (85/729) of greyhounds returned within six months. This appears to suggest 392 greyhounds are less likely to be returned within one month than dogs rehomed from a 393 shelter, but that greyhounds have a similar six-month post-adoption return rate to 394 shelter dogs.

396 Most greyhound returns occurred within four months of adoption, the first peak was 397 around one month post-adoption (22\% of returns), but a second peak occurred around 398 three months post-adoption, with $33.1 \%$ of returns between one and six months post399 adoption. In contrast, Shore (2005) reported the majority of shelter returns (56\%) were 400 within one month of adoption, and only $20 \%$ were between one and six months. Elliott 
401 et al (2010) reported the types of behaviour problems, associated with increased risk of 402 greyhound adoption failure within one month, were similar (albeit at a lower incidence)

403 to those reported in shelter studies (i.e. separation anxiety, noisiness, aggression 404 towards children, problems with existing pets). Given the greyhound return rate 405 increases to match that of shelter studies at 6 months, it would be interesting to 406 investigate factors associated with increased risk of return as adoption time increases.

\section{Dog characteristics associated with rehoming success}

Our data show young greyhounds are more likely to pass the temperament test than 411 adult or senior dogs. When we considered the dogs that were potentially available for 412 adoption (i.e. had passed the temperament test) there was no detectable age effect on 413 rehoming success, either at first adoption, or ultimately (at second or subsequent 414 adoptions).

416 It would appear that although young greyhounds are less likely to fail the temperament 417 test, they are no less likely to be returned following adoption than older dogs. Because 418 the GAP temperamant test has not been scientifically tested for validity and reliability, 419 we cannot rule out the possibility the detected age effect is a result of bias within the 420 temperament test design or application. Although other studies have shown an 421 association between age, and the type and prevalence of problem behaviours in 422 rehomed dogs (Wells \& Hepper 2000), there appears to be no association between age 423 and increased risk of failed adoption (Diesel et al 2008b, Elliott et al 2010, Wells \& 424 Hepper 2000). 
427 The literature suggests that younger dogs tend to show a greater number of problematic 428 behaviours than older dogs (Blackwell et al 2008), but the types of problems are 429 significantly different (Blackwell et al 2008; Wells \& Hepper 2000). Younger dogs are 430 more likely to have control problems and display separation-related and attention 431 seeking behaviours, but are less likely than older dogs to show undesirable behaviours 432 associated with aggression, reactions to other dogs and unfamiliar people (Blackwell et 433 al 2008; Wells and Hepper 2000). Demonstrating aggressive reactions to other dogs or 434 unfamiliar people would cause a dog to fail the temperament test but attention seeking 435 or separation related behaviours may not. However, all of these behaviour problems are 436 associated with increased risk of failed adoptions (Diesel et al 2008b, Elliott et al 2010, 437 Wells \& Hepper 2000), supporting our loss of an age effect post-adoption.

439 The other detectable influence on ultimate rehoming success was sex of dog, with male 440 dogs being less likely than females to be successfully rehomed. Although more females 441 passed the temperament test with a TTP+ than males, females were no more likely to 442 be successfully adopted at first adoption (i.e. no less likely to be returned) than males. 443 Although Wells \& Hepper (2000) reported male dogs were more likely to demonstrate 444 behaviour problems associated with increased risk of failed adoption, most of the dogs 445 in their study were entire and it was suggested the reported behaviour problems 446 (aggression towards other dogs, sexual behaviours and straying) were likely to be under 447 hormonal control. In contrast, all greyhounds are desexed prior to rehoming. Elliott et 448 al (2010) identified differences in the type of behaviour problems likely to be displayed 449 by recently adopted male greyhounds compared with females (i.e. males more likely to 450 show aggression towards small dogs, cats and when approached on bed, cf. females 
451 more likely to show destructivness in the yard), but, consistent with our study, did not

452 find sex a risk factor associated with post-adoption return. Other studies have found no 453 association between sex and risk of return, and either the type or prevalence of 454 behaviour problems in rehomed dogs (Blackwell et al 2008; Diesel et al 2008b).

456 The reasons greyhounds are fostered prior to adoption vary, but generally it is to 457 evaluate the dogs ability to transition to pet life whilst providing them with support to 458 overcome challenges often associated with socialisation deficits and long term 459 kennelling (Elliott et al 2010). These challenges include fear of unfamiliar 460 environments, sounds, people and animals. There is no standardised foster period or 461 process, but just over a third of greyhounds entering GAP are fostered prior to adoption. 462 Although there is no documented criteria for selecting dogs to foster, there is a 463 suggestion dogs displaying borderline behaviours upon entering GAP, or during their 464 temperament assessment, are more likely to be fostered. For example, the temperament 465 test (see methods) suggests dogs not reaching the adoption standard should be fostered 466 where their behaviour is borderline, and further, $56.1 \%$ of fostered dogs had passed the 467 temperament test with a basic TTP compared with $38.8 \%$ of dogs that were directly 468 adopted (a TTP result was shown to be associated with decreased likelihood of 469 successful adoption overall, compared to dogs achieving TTP+). Despite the potential 470 bias in selecting dogs for foster that might have more difficulty in being successfully 471 rehomed, $14.4 \%$ of fostered dogs were returned from adoption compared with $18.1 \%$ 472 of dogs that were not fostered first, suggesting that fostering contributes to rehoming 473 success. Investigation of the criteria, methods and effects of fostering would be 474 necessary to identify elements associated with adoption success and aid standardisation 475 of the process. 
477 Trainers do not all raise, manage or train their dogs in the same way, and some authors report on an extremely wide range of practices (Atkinson \& Young 2005; Huggins

479 2007), which have the potential to impact on the adoptability of dogs. Our evaluation 480 of trainer effects is largely based on descriptive analysis due to the small subset of data 481 restricted to the five trainers entering $25 \mathrm{dogs}$ or more into the GAP programme. 482 Because of this small subset, we were unable to explore trainer effects on rehoming 483 success of adopted dogs. From our descriptive analyses, there were observed 484 differences among trainers in entry reason, sex and age profile of dogs entered, 485 suggesting differences in the criteria used to select dogs to enter the adoption 486 programme. For example, two trainers entered considerably more younger dogs than 487 the others. Although our previous analysis showed younger dogs were more likely to 488 pass the temperament test than older dogs, our data show no significant effect of trainer 489 on temperament test outcome.

\section{Conclusion}

493 Greyhound adoption is very successful for those dogs entering the GAP programme. 494 Younger dogs were more likely to pass the temperament test, possibly due to having 495 more malleable temperaments, and having their predatory behaviour reinforced for less 496 time than older adults involved in training and racing for more than 2 years. However, 497 this observed age effect might also reflect an unjustified bias in either the design or 498 application of the temperament test, as the success of adopted dogs was not affected by 499 age. Sex (female) and temperament test outcome (TTP+) were factors associated with 
500 increased adoption success and warrant further investigation in terms of the type and

501 prevalence of behaviour problems that place greyhound adoptions at risk.

503 Further understanding of factors likely to impact greyhound adoption are likely to be

504 obscured because most dogs do not enter the programme and, for those that do, there is

505 a lack of clarity around the criteria used by trainers in their selection. While we 506 appreciate the practical necessity of having the temperament test to assess the suitability 507 of dogs for adoption, this additional removal of dogs from the adoption process 508 provides another unavoidable bias in our sample population. Testing the range of 509 effects on successful adoption is restricted to those dogs passing an as yet scientifically 510 unvalidated temperament test. Although the GAP temperament test showed reliability 511 over time, the programme would benefit from scientfic evaluation of the validity and 512 reliability of the temperament test.

\section{Animal welfare implications}

515 Although a small percentage (14.5\%) of greyhounds entering GAP fail to be 516 successfully rehomed, the vast majority (80\%) of those that fail, fail the entry 517 temperament test, which is as yet scientifically unvalidated. The possibility of 518 unjustified age bias in either the temperament test design or implementation could 519 affect the number of adult dogs (> 24 months old) that are destroyed before being made 520 available for adoption. 


\section{References}

Atkinson M and Young K 2005 Reservoir dogs: Greyhound racing, mimesis and sports-related violence. International Review for the Sociology of Sport, 40(3): 335$356+394+397+400$

Bennett SL, Litster A, Weng HY, Walker, SL, and Luescher AU 2012 Investigating behavior assessment instruments to predict aggression in dogs. Applied Animal Behaviour Science, 141(3-4), 139-148

Blackwell EJ, Twells C, Seawright A, and Casey RA 2008 The relationship between training methods and the occurrence of behavior problems, as reported by owners, in a population of domestic dogs. Journal of Veterinary Behavior: Clinical Applications and Research, 3(5): 207-217

Colgan B, Neil C, and Foy L 2013 New Zealand Greyhound Racing Association, Independant Welfare Review (pp 1-56). New Zealand: WHK

Coren S 2000 Why we love the dogs we do. New York, USA: Fireside, Simon \& Schuster Inc.

Diesel G, Smith H, and Pfeiffer DU 2007 Factors affecting time to adoption of dogs re-homed by a charity in the UK. Animal Welfare, 16(3), 353-360.

Diesel G, Brodbelt D, and Pfeiffer DU 2008a Reliability of assessment of dogs' behavioural responses by staff working at a welfare charity in the UK. Applied Animal Behaviour Science, 115(3-4): 171-181

Diesel G, Pfeiffer DU, and Brodbelt D 2008b Factors affecting the success of rehoming dogs in the UK during 2005. Preventive Veterinary Medicine 84(3-4): 228241

Duffy DL, Hsu Y, and Serpell JA 2008 Breed differences in canine aggression. Applied Animal Behaviour Science, 114(3-4): 441-460

Elliott R, Toribio JALML, and Wigney D 2010 The Greyhound Adoption Program (GAP) in Australia and New Zealand: A survey of owners' experiences with their greyhounds one month after adoption. Applied Animal Behaviour Science, 124(3-4): 121-135

Fogle B 2000 The new encyclopedia of the dog (Second ed.). New York, USA: Dorling Kindersley Publishing Inc.

GAP 2014 Greyhounds As Pets Annual Report (Year ending 31 March 2014 ed.). Wellington, New Zealand

Huggins M 2007 Betting, sport and the British, 1918-1939. Journal of Social History, 41(2): 283-306

Lord LK, Yaissle JE, Marin L, and Couto CG 2007 Results of a web-based health survey of retired racing greyhounds. Journal of Veterinary Internal Medicine, 21(6): 1243-1250

Madden R 2010 Imagining the greyhound: 'Racing' and 'rescue' narratives in a human and dog relationship. Continuum, 24(4): 503-515 Marder AR, Shabelansky A, Patronek GJ, Dowling-Guyer S, and D'Arpino SS 2013 Food-related aggression in shelter dogs: A comparison of behavior identified by 
564 a behavior evaluation in the shelter and owner reports after adoption. Applied Animal 565 Behaviour Science, 148(1-2), 150-156

566 Marston LC and Bennett PC 2003 Reforging the bond-towards successful canine 567 adoption. Applied Animal Behaviour Science, 83(3): 227-245

568 Marston LC, Bennett PC and Coleman GJ 2004 What happens to shelter dogs? An 569 analysis of data for 1 year from three Australian shelters. Journal of Applied Animal $570 \quad$ Welfare Science, 7(1): 27-47

571 Marston LC, Bennett PC and Colemon GJ 2005 Adopting shelter dogs: Owner 572 experiences of the first month post-adoption. Anthrozoos, 18(4): 358-378

573 McGreevy PD, and Masters AM 2008 Risk factors for separation-related distress and 574 feed-related aggression in dogs: Additional findings from a survey of Australian dog 575 owners. Applied Animal Behaviour Science, 109(2-4), 320-328

576 McMillan FD, Duffy DL and Serpell JA 2011 Mental health of dogs formerly used 577 as 'breeding stock' in commercial breeding establishments. Applied Animal Behaviour 578 Science, 135(1-2): 86-94

579 Miklósi Á 2007 Dog Behaviour, Evolution, and Cognition. New York: Oxford 580 University Press.

581 Mornement KM, Coleman GJ, Toukhsati S and Bennett PC 2010 A review of 582 behavioral assessment protocols used by australian animal shelters to determine the 583 adoption suitability of dogs. Journal of Applied Animal Welfare Science, 13(4): 314$584 \quad 329$

585 Mornement KM, Coleman GJ, Toukhsati SR, and Bennett PC 2015 Evaluation of 586 the predictive validity of the Behavioural Assessment for Re-homing K9's (B.A.R.K.) 587 protocol and owner satisfaction with adopted dogs. Applied Animal Behaviour Science, $588 \quad 167,35-42$

589 Paroz C, Gebhardt-Henrich SG and Steiger A 2008 Reliability and validity of 590 behaviour tests in Hovawart dogs. Applied Animal Behaviour Science 115(1-2): 67-81

591 Patronek GJ, Glickman LT, and Moyer, MR 1995 Population Dynamics and the 592 Risk of Euthanasia for Dogs in an Animal Shelter. Anthrozoos, 8(1), 31-43

593 Rooney NJ, Gains SA and Bradshaw JWS 2007 Behavioural and glucocorticoid 594 responses of dogs (Canis familiaris) to kennelling: Investigating mitigation of stress by 595 prior habituation. Physiology and Behaviour, 92(5): 847-854

596 Seksel K, Mazurski EJ and Taylor AH 1999 Puppy socialisation programs: short and 597 long term behavioural effects. Applied Animal Behaviour Science, 62(4): 335-349

598 Shore ER 2005 Returning a recently adopted companion animal: Adopters' reasons for 599 and reactions to the failed adoption experience. Journal of Applied Animal Welfare 600 Science 8(3): 187-198

601 Taylor, KD and Mills, DS 2006 The development and assessment of temperament 602 tests for adult companion dogs. Journal of Veterinary Behavior: Clinical Applications 603 and Research, 1(3): 94-108

604 Taylor KD and Mills DS 2007 The effect of the kennel environment on canine welfare:

605 A critical review of experimental studies. Animal Welfare, 16(4): 435-447 
606 Thompson KF, McBride EA and Redhead E 2010 Training engagement and the 607 development of behaviour problems in the dog: A longitudinal study. Journal of 608 Veterinary Behaviour: Clinical Applications and Research, 5(1): 57

609 Wells DL 2004 A review of environmental enrichment for kennelled dogs, Canis 610 familiaris. Applied Animal Behaviour Science, 85(3-4): 307-317

611 Wells DL, Graham L and Hepper PG 2002 The influence of length of time in a rescue 612 shelter on the behaviour of kennelled dogs. Animal Welfare, 11(3): 317-325

613 Wells DL and Hepper PG 2000 Prevalence of behaviour problems reported by owners 614 of dogs purchased from an animal rescue shelter. Applied Animal Behaviour Science, 615 69(1): 55-6555

616 Žák J, Voslářová E, Večerek V and Bedáňová I 2015 Sex, age and size as factors 617 affecting the length of stay of dogs in Czech shelters. Acta Veterinaria Brno, 84(4), $618 \quad 407-413$ 